\title{
La Transición a la Vida Activa de las Personas con Discapacidad: Expectativas Familiares y Grado de Ajuste al Trabajo ${ }^{1}$ TRANSition to Active Life of PeOple With Disabilities: Family EXPECTATIONS AND DEGREE OF WORK ADJUSTMENT
}

\author{
Ana RIAÑO-GALÁN ${ }^{2}$ \\ Alejandro RODRÍGUEZ-MARTÍN ${ }^{3}$ \\ Rosa GARCÍA-RUIZ ${ }^{4}$ \\ Emilio ÁLVAREZ-ARREGUI ${ }^{5}$
}

\begin{abstract}
RESUMO: as pessoas com deficiência física ou mental têm direito a ser inseridas no mercado de trabalho para exercer seu papel como cidadáos ativos, independentes e com um nível de qualidade de vida adequado, o que constitui um autêntico desafio ao qual devemos responder. No caso deste estudo, nos limitamos ao tema deficiência intelectual. No que diz respeito ao âmbito educativo, os Programas de Qualificação Profissional Inicial (PCPI), na modalidade de Aula de Formação Específica, oferecem una formação adequada ao patamar de competências sociais, pessoais e laborais para este coletivo. Neste trabalho, apresentamos os resultados da aplicação de um Itinerário de Inserção Laboral, dentro do quadro de um projeto europeu, no qual participaram estudantes de três centros educativos da Finlândia, Espanha e Dinamarca, assim como as suas respetivas famílias. Para conhecer o grau de ajustamento ao trabalho destes jovens, estes responderam ao Work Adjustment Inventory-WAI (Inventário de Ajustamento ao Trabalho) e também entrevistamos as suas famílias. Os resultados permitiram saber que o itinerário de inserção laboral desenhado constitui uma oferta formativa adequada para os estudantes em questão, enfatizamos que o grau de adaptabilidade ao trabalho está relacionado com o nível de atividade, empatia, sociabilidade e assertividade de cada pessoa. As entrevistas possibilitaram a identificação do perfil das famílias destes estudantes e identificar as suas expectativas em relação ao futuro dos seus filhos e filhas. Entre as conclusôes alcançadas, destacam-se as altas expectativas das famílias no que diz respeito à inserção dos seus filhos, assim como o alto nível de avaliaçáo relativamente ao itinerário desenhado como estratégia fundamental para conseguir lograr uma formação integral e uma inserção sócio-laboral adequada.
\end{abstract}

PALAVRAS-CHAVE: Instituiçôes de Educaçẫo Especial. Relação Educação/Trabalho. Educação Especial. Modalidades de Educação Profissional. Educação de Jovens e Adultos.

\begin{abstract}
People with mental or physical disabilities have the right to join the labour market in order to be active, independent citizens with a sufficient standard of living, which is a challenge that must be met. In this paper we discuss the theme of intellectual disability. In terms of education, the Specialised Workshops included in Initial Professional Qualification Programmes (PCPI for its initials in Spanish) offer suitable training in social, personal and work skills for this group of people. The results of applying a Job Placement Itinerary within a European project framework, which involved students from three schools in Finland, Spain and Denmark as well as their families, are presented in this paper. To measure the degree of work adjustment these young people have responded to the Work Adjustment Inventory-WAI and their families were interviewed. The results have determined that the designed job placement itinerary constitutes a suitable training option for these students and emphasized that the degree of work adjustment is related to the individual's level of activity, empathy, sociability and assertiveness. Through the interviews it was possible to identify the profile of these students' families and their expectations about the future of their children. The final conclusions underscore the high expectations of families for the inclusion of their children, as well as the high value placed on the designed itinerary as a key strategy for more comprehensive training and suitable social and labour integration.
\end{abstract}

KEYWORDS: Institutions of Special Education. Education-Work Relations. Special Education. Methods of Professional Education. Youth and Adult Education.

\footnotetext{
${ }^{1}$ Este artículo se ha realizado en el marco de la investigación del Proyecto Europeo Teaching in Competences. A step towards inclusion (código 2009-1-ES1-GRU06-09719) financiado por la Unión Europea a través del Lifelong Learning Programme (iniciativa Grundtvig).

${ }^{2}$ Doctora en Psicopedagogía; Centro de Ed. Especial Dr. Fernando Arce , Cantabria, Espańa. rianoam@gmail.com

${ }_{3}^{3}$ Doctor en Psicopedagogía, Departamento de Ciencias de la Educación de la Universidad de Oviedo, Asturias, España. rodriguezmalejandro@uniovi.es

${ }^{4}$ Doctora en Psicopedagogía, Departamento de Educación de la Universidad de Cantabria, Cantabria, España. rosa.garcia@unican.es

${ }^{5}$ Doctor en Pedagogía, Departamento de Ciencias de la Educación de la Universidad de Oviedo, Asturias, España.

alvarezemilio@uniovi.es
} 


\section{INTRODUCCIÓN}

La evolución de la investigación en el ámbito de la Educación Especial en Europa se ha caracterizado por centrarse en temas concretos como el diagnóstico y el tratamiento de las diferentes discapacidades, situando las teorías y modelos psicológicos en el marco de referencia temporal. Tradicionalmente, el énfasis se ponía en la discapacidad y el binomio diagnósticotratamiento/programa. Sin embargo, en estos momentos, la evolución de los intereses de la investigación se centra en la mejora de la escuela en su conjunto, desde los supuestos de la Educación Inclusiva que promueven la atención y respuesta a todo el alumnado en las mismas escuelas y clases. Este enfoque repercute en la investigación porque amplía su espectro de análisis a las interacciones que se producen en distintos contextos simultáneamente. Se incrementa pues, el interés hacia las consecuencias sociales y subjetivas e intersubjetivas de la discapacidad, contemplando su significado con respecto a un contexto social más amplio y se observa, desde la práctica, una gran necesidad de ampliar el ámbito de la investigación desde lo escolar hacia al empleo y el logro de una vida independiente para las personas con discapacidad; lograr calidad de vida.

En este escenario no se puede obviar que el trabajo constituye un derecho para todas las personas y así se proclama en la Declaración Universal de los Derechos Humanos de $1948^{6}$ (art. 23) y la Carta de los derechos fundamentales de la Unión Europea ${ }^{7}$ (art. 23). Sin embargo, este derecho no está generalizado, más aún en una situación de crisis económica global que ha hecho retroceder las tasas de empleo a límites de hace décadas, aumentando de manera exponencial las cifras de desempleo en muchos países con las negativas consecuencias que ello implica.

Esta situación se agrava aún más en las personas con discapacidad pues, a pesar del reconocimiento de derechos y del conjunto de medidas impulsadas para promover la inserción laboral de este colectivo, existe un conjunto de dificultades específicas para formarse laboralmente, encontrar y mantener un puesto de trabajo (Pallisera, Vilá y Valls, 2003). Ello exige el respeto a sus diferencias y un esfuerzo por ofertar oportunidades reales que permitan la realización de proyectos de vida propios que promuevan su transición a la vida adulta mediante la adquisición de competencias claves que favorezcan su inserción laboral desde una visión inclusiva de la sociedad y la persona (Carnaby y Lewis, 2005; Pallisera, 2010; Schalock 2013).

\subsection{LA ATENCIÓN A LA DIVERSIDAD EN JÓVENES DE EDUCACIÓN SECUNDARIA OBLIGATORIA Y POST-OBLIGATORIA}

La extensión de la educación especial al alumnado con altas capacidades, dificultades específicas de aprendizaje, problemas de inadaptación social o atención a las minorías étnicas ha generado que la concepción tradicional del término haya ido evolucionando, haciendo emerger nuevos términos como atención a la diversidad, comprensividad o educación inclusiva, etc.

La atención a la diversidad introduce un concepto más amplio, puesto que no se circunscribe sólo a la educación especial, que tradicionalmente se ha centrado en la discapacidad. La diversidad abarca dimensiones culturales, lingüísticas, de acceso al conocimiento, sociales, de género, de necesidades educativas especiales asociadas a discapacidades, trastornos de

${ }^{6}$ Disponible en: https://www.un.org/es/documents/udhr/

${ }^{7}$ Disponible en: http://www.europarl.europa.eu/charter/pdf/text_es.pdf 
aprendizaje y altas capacidades intelectualmente entre otros. Sin embargo, el término diversidad sigue teniendo un sentido reducido porque se aplica al alumnado que significativamente está por debajo del rendimiento estándar del alumnado o aparece asociado a prácticas educativas ligadas a alumnos con déficit y que requieren respuestas distintas a las que señala el currículo ordinario.

La escuela inclusiva, por el contrario, define la educación centrada en la atención a las necesidades individuales de todo el alumnado, partiendo de que los centros, como comunidades educativas, deben satisfacer las necesidades del mismo, independientemente de que sus diferencias sean personales o sociales. (Stainback y Stainback, 1999).

En el caso de España y gracias a las Adaptaciones Curriculares Individuales (ACI) y otros medidas de atención a la diversidad, la respuesta educativa que se da al alumnado con necesidades específicas de apoyo educativo se ha ido incrementando y enriqueciendo desde los ańos noventa, con la publicación de la Ley Orgánica 1/1990, de 3 de octubre de 1990, de Ordenación General del Sistema Educativo (LOGSE'), y las sucesivas leyes que han regulado el sistema educativo, como la Ley Orgánica 2/2006, de 3 de mayo, de Educación (LOE ${ }^{9}$ ) y la actual Ley Orgánica 8/2013, de 9 de diciembre, para la mejora de la calidad educativa $\left(\right.$ LOMCE $\left.^{10}\right)$.

Sin embargo, esta propuesta ha tenido mayor acogida en la etapa de Educación Primaria que en la Educación Secundaria donde el fracaso académico muestra cifras alarmantes situándolo en objetivo de numerosos estudios (Eckert, 2006; Calero, Choi y Waisgrais, 2010; Suárez et al., 2011) que analizan su alto impacto, identificando la existencia de condicionantes como la implicación familiar, los rígidos planteamientos del sistema educativo, las características de los centros o las propias del alumnado.

En este marco es donde surgen con la LOE y los Programas de Cualificación Profesional Inicial (PCPI). Su finalidad es que todo el alumnado que los curse alcancen competencias profesionales propias de una cualificación de Nivel 1 de la estructura actual del Catálogo Nacional de Cualificaciones Profesionales creado por la Ley 5/2002, de 19 de junio (LCUFP ${ }^{11}$ ), así como que tengan la posibilidad de una inserción sociolaboral satisfactoria.

El alumnado que los cursa suele tener dificultades para adaptarse a los objetivos de su nivel de edad, debido a las fuertes lagunas que arrastran de una escolaridad muy irregular. Presentan características y ritmos de aprendizaje distintos y diversidad de motivaciones y necesidades; baja autoestima y baja eficacia para afrontar cualquier tarea que guarde relación con la escuela. Suelen tener una vinculación negativa con el centro educativo, motivada por los constantes conflictos curriculares y disciplinarios y, en muchas ocasiones, carecen de apoyo e implicación familiar.

Todas estas variables provocan que el tránsito entre la vida académica y la vida laboral de este conjunto de jóvenes sea especialmente complejo, teniendo en cuenta que pueden ser considerados alumnos en riesgo puesto que encuentran, a lo largo de su escolaridad

\footnotetext{
${ }^{8}$ LOGSE. Disponible en: http://www.boe.es/buscar/doc.php?id=BOE-A-1990-24172

${ }^{9}$ LOE. Disponible en: http://www.boe.es/buscar/doc.php?id=BOE-A-2006-7899

${ }^{10}$ LOMCE. Disponible en: https://www.boe.es/diario_boe/txt.php?id=BOE-A-2013-12886

${ }^{11}$ LCUFP. Disponible en: http://www.boe.es/buscar/act.php?id=BOE-A-2002-12018
} 
y en determinados momentos críticos de la misma, dificultades acusadas para seguir con aprovechamiento el currículo y la educación general.

Por tanto, los planteamientos actuales van dirigidos a que el alumnado con necesidades educativas especiales y/o problemas de aprendizaje reciba un apoyo adicional, no tanto en contenidos curriculares inespecíficos o descontextualizados; sino planificada desde una formación centrada en estrategias de enseñanza-aprendizaje ligadas a la comprensión de textos, mejora de la comunicación oral y de la expresión escrita, dominio del cálculo, habilidades sociales, habilidades emocionales y aprendizajes en entornos vitales y profesionales, todo ello encaminado al desarrollo de sus competencias personales, sociales y laborales.

\subsection{INCLUSIÓN SOCIOLABORAL DE JÓVENES CON DISCAPACIDAD: LA RESPUESTA DE LOS P.C.P.I.}

Los Programas de Cualificación Profesional Inicial (PCPI) constituyen una de las novedades más importantes de la ya extinta LOE implantadas durante el curso 2008-2009. La finalidad clave de los PCPI es que el alumnado tenga la posibilidad de una inserción sociolaboral satisfactoria que da la oportunidad de obtener las competencias profesionales propias de una cualificación profesional de nivel 1.

Los PCPI se diseñan como una vía más para favorecer la inserción social, educativa y laboral de los jóvenes mayores de 16 años que no hayan obtenido el título de Graduado en Educación Secundaria Obligatoria. Permiten al alumnado la posibilidad de una salida profesional homologada, la oportunidad de superar la prueba de acceso a Ciclos Formativos de Grado Medio de Formación Profesional y la posibilidad de obtener el título de Graduado en Educación Secundaria.

En el caso de los jóvenes con discapacidad, los PCPI suponen una medida de atención a la diversidad clara y fundamental. Su finalidad es ofrecer una vía alternativa al alumnado con necesidades educativas especiales para la inserción laboral, en puestos de trabajo acordes con su discapacidad en centros especiales de empleo o en centros ocupacionales, tanto para trabajo normalizado como para trabajo con apoyo ${ }^{12}$.

Las investigaciones centradas en la discapacidad y el mundo laboral son abundantes aunque desde muy diversos enfoques. Entre las más destacadas encontramos los trabajos de Vilá y Pallisera (2006) sobre el proceso de integración laboral seguido por personas con discapacidad intelectual límite o la de Gorostidi y Navarro (2007), respecto a la inserción laboral de las personas con discapacidad mediante el empleo con apoyo. El estudio de Montes Guidox (2007) es importante ya que analiza la realidad de las familias con hijos con discapacidad a la hora de conciliar la vida familiar y laboral. También destacan las investigaciones sobre la relación escuela-trabajo que comienzan a ser particularmente numerosas en los últimos años. Se refieren a programas de transición entre la escuela y la vida adulta (Sánchez y Jurado, 2001; León, 2001) y a experiencias de empleo con apoyo (Verdugo y Jordán, 1998; Sanahuja, Núñez y Jurado, 2001).

\footnotetext{
${ }^{12}$ El Trabajo con Apoyo o el Empleo con Apoyo consiste en un conjunto de servicios y acciones centradas en la persona, fundamentalmente individualizadas, para que la persona con discapacidad y con especiales dificultades pueda acceder, mantenerse y promocionarse en una empresa ordinaria en el mercado de trabajo abierto, con el apoyo de profesionales y otros tipos de apoyos.
} 
Asimismo, y centrados en los Programas de Cualificación Profesional Inicial, cabe mencionar el estudio de Merino, García y Casal (2006) que hace un balance de lo que han representado los PCPI y la articulación de los recursos locales para la inserción laboral. Otros estudios importantes, como los desarrollos por Vega y Aramendi (2010, 2011), concluyen que los PCPI constituyen una medida orientada a la inserción sociolaboral de los jóvenes con discapacidad pero que requiere en su desarrollo una atención educativa integral del alumnado que atienda a la formación laboral y también a las limitaciones físicas, intelectuales, culturales, emocionales y sociales de los propios participantes. Estos autores también subrayan que los PCPI requieren de un currículo más funcional e integrador que se oriente a unidades de aprendizaje más globales y una metodología específica propia estrechamente vinculada con la plena inserción social y laboral. A este respecto cabe indicar que las investigaciones sobre los PCPI adaptados (o modalidad Taller especifico) destinados a personas con discapacidad son más escasas.

La modalidad de PCPI adaptado o Taller Especifico se desarrolla en centros de educación especial o entidades sin ánimo de lucro y constituye, por tanto, un elemento clave en la trayectoria vital de los jóvenes con discapacidad porque debe ayudarles a construir un itinerario formativo que satisfaga sus demandas personales y promueva su inserción sociolaboral integrando de manera flexible tres aspectos: Formativo, Psicosocial y Laboral, que interactúan entre sí durante todo el itinerario personalizado de inserción que se implemente.

De manera general, con el concepto itinerario personalizado de inserción se hace alusión a un determinado modelo metodológico, capaz de ofrecer una respuesta integradora a los diferentes recursos y servicios, destinado a las personas que, por diferentes motivos, presentan dificultades especiales para incorporarse al mundo laboral y necesitan un servicio eficaz y adecuado a sus características que compense diferencias y haga efectivo el principio de igualdad de resultados en el proceso de inserción laboral de jóvenes con discapacidad.

Desde estos referentes, el objetivo de este estudio que presentamos es doble. Por un lado, analizar el ajuste al trabajo del alumnado participante en el itinerario personalizado de inserción en los tres centros de España, Dinamarca y Finlandia y, por otro lado, conocer las expectativas de futuro que sobre su inserción laboral tienen las respectivas familias de estos jóvenes con discapacidad.

\section{Método}

El diseño elegido para la investigación ha sido no experimental (Bisquerra, 2004) y se ha centrado en los análisis de casos (Cook y Reichardt, 2005; Stake, 2008) de los tres centros educativos europeos mencionados, prestando especial atención al grado de ajuste al trabajo del alumnado participante y a las expectativas familiares en relación a la inserción sociolaboral de los alumnos de dichos centros. Dado que estos tres centros aplican metodologías de intervención diferentes, se procedió a realizar análisis cruzados y tratando de complementar e integrar la perspectiva metodológica cuantitativa y la cualitativa (Flick, 2004). 


\subsection{Participantes}

Los participantes que constituyen la muestra de este estudio, siguiendo el ciclo de muestreo propuesto por Fox (1981), se han tomando de los grupos naturales de alumnos/ as de entre 16 y 25 años con necesidades educativas especiales $(\mathrm{N}=26)$ que están inscritos en programas formativos en los tres centros europeos participantes en la investigación dentro del Proyecto Europeo Teaching in Competences. A step towards inclusion (Asociación Grundtvig):

- Karkun Evankelinen Opisto (Karkku-Finlandia).

- Bronderslev Educationcenter (Bronderslev-Dinamarca).

- $\quad$ Centro de Educación Especial Dr. Fernando Arce (Cantabria-España).

La distribución de la muestra y sus características en cuanto a género y nacionalidad quedó finalmente configurada tal y como se presenta en la Tabla 1.

Tabla 1 - Participantes

\begin{tabular}{l|c|c|c}
\hline País & Hombre & Mujer & Total \\
\hline Finlandia & 8 & 0 & 8 \\
\hline Dinamarca & 8 & 0 & 8 \\
\hline España & 5 & 5 & 10 \\
\hline Total & $\mathbf{2 1}$ & $\mathbf{5}$ & $\mathbf{2 6}$ \\
\hline
\end{tabular}

Fuente: Elaboración propia

En el caso de España nos encontramos con una muestra de 10 personas con distinto tipo y grado de discapacidad ( 5 chicos y 5 chicas). En Finlandia y en Dinamarca han participado un total de 16 alumnos (8 daneses y 8 finlandeses) con necesidades específicas por diversos motivos. Se trata, por consiguiente, de un procedimiento de muestreo no probabilístico, por lo que las conclusiones extraídas de la investigación han atendido preferentemente a la validez interna.

En cuanto a las entrevistas realizadas a las familias $(\mathrm{N}=23)$, se citaron a ambos cónyuges en todos los casos que fue posible, si bien las entrevistas se realizaron individualmente en el $90 \%$ de los casos ya que únicamente acudía la madre.

\subsection{INSTRUMENTOS Y PROCEDIMIENTOS}

La investigación en la que se enmarca el estudio que aquí se presenta se ha llevado a cabo en dos fases principales: una de análisis de casos y otra pedagógica-aplicada. En la primera, la aplicación de las diversas técnicas para la recogida de datos (cuestionario y entrevista) ha dado lugar a un estudio analítico-descriptivo de los tres grupos en los centros europeos participantes. La segunda fase, de carácter descriptivo-interpretativo, ha partido del análisis de los resultados relevantes obtenidos en la primera fase y ha dado lugar al diseño del itinerario de inserción laboral recogido en la Tabla 1 y que se continúa implementando actualmente.

En cuanto a los instrumentos seleccionados, atendiendo al primer objetivo del estudio centrado en conocer el grado de ajuste al trabajo del alumnado de los centros europeos 
participantes, se ha empleado el Work Adjustment Inventory-WAI (Inventario de Ajuste al Trabajo) (Gilliam, 1994). Esta prueba evalúa el ajuste del alumnado de nuestra muestra al mundo laboral a partir de seis escalas (actividad, empatía, sociabilidad, asertividad, adaptabilidad y emocionalidad).

Este instrumento se aplicó en su formato original en inglés en los colegios de Finlandia y Dinamarca. Sin embargo, en el caso de España se procedió a su traducción al castellano. Para garantizar las propiedades psicométricas del instrumento, los pasos seguidos, en términos generales, consistieron en: 1) la traducción de ítems, 2) la valoración por parte de jueces expertos, 3) su aplicación piloto, 4) el análisis de ítems, discriminación y fiabilidad, 5) el análisis de la escala, validez y fiabilidad, y 6) el análisis factorial, para contrastar su validez de constructo. Los resultados obtenidos avalan la capacidad discriminativa, la fiabilidad y la validez del WAI, en su versión adaptada al castellano.

Los análisis de datos del cuestionario fueron realizados mediante el paquete estadístico SPSS 19.0 obteniendo un análisis de estadísticos descriptivos y correlaciones entre las escalas del WAI.

Para abordar el segundo objetivo del estudio, se diseñó una entrevista semiestructurada que permitió tener una visión global del conocimiento y expectativas de las familias hacia sus hijos/ as con discapacidad. Para ello se plantearon una treintena de cuestiones relativas a tres ámbitos:

- $\quad$ momento y modo en que las familias recibieron la información de la discapacidad del hijo/a e identificación de las competencias actuales del hijo/a en actividades domésticas.

- $\quad$ expectativas ante la discapacidad en el entorno familiar y proyección de las expectativas generales hacia el hijo/a.

- $\quad$ expectativas sobre el alcance futuro de la discapacidad en diferentes momentos de la vida.

Tras contactar con las familias y solicitar su disponibilidad para ser entrevistadas se acordaron las entrevistas en función de las preferencias horarias y disponibilidad. Se informó de los objetivos de la investigación, de la confidencialidad de la información obtenida y se solicitó autorización para la grabación de toda la entrevista y su posterior procesamiento.

El procedimiento de análisis sigue las directrices de Taylor y Bogdan (2000) para la investigación cualitativa: a) descubrimiento, d) codificación y c) relativización para buscar una mayor comprensión y profundidad en el análisis de la realidad de los estudiantes objeto de estudio.

En cuanto a la construcción del sistema de categorías y de códigos nos hemos apoyado en la propuesta de Miles y Huberman (1994) y en el programa AQUAD 6.0. Así, se han acomodado las transcripciones a las exigencias del software, posteriormente se ha realizado un análisis de contenido codificando segmentos de significado y agrupando las codificaciones en catálogos y categorías que cumplen las tres condiciones básicas reconocidas: ad hoc, exhaustividad y mutua exclusividad. La información proporcionada por las entrevistas supone más de 70 horas de grabación sobre diferentes ámbitos. 
Para evitar el sesgo de expectancia y las posibles inconsistencias del entrevistador, las codificaciones se han validado, en diferentes momentos, a través de cuatro revisiones internas de profesores de las dos universidades participantes y, también, de una revisión de cuatro expertos de la Universidad de Cantabria y dos de la Universidad de Oviedo. Se ha medido la concordancia en función del grado de acuerdo en el registro a través del coeficiente de Kappa de Cohen obtenido mediante el SPSS y que arroja un alto nivel de concordancia ( $\leq \leq .00)$. Asimismo, para guardar el rigor metodológico y procedimental, se han seguido los criterios de calidad científica propuestos por autores como Merterns (2005) o Franklin y Ballau (2005) en cuanto a dependencia, credibilidad, auditabilidad y transferibilidad.

\section{Resultados}

A continuación se presentan los principales resultados obtenidos tras la aplicación del Work Adjustment Inventory-WAI y la realización de las diferentes entrevistas a las familias del alumnado participante.

\subsection{EXPECTATIVAS DEL ALUMNADO CON DISCAPACIDAD SOBRE SU GRADO DE AJUSTE AL TRABAJO}

Tras la aplicación Work Adjustment Inventory-WAI y el procesamiento de los datos obtenidos en cada país, se obtuvieron los datos descriptivos de la muestra que se recogen en la Tabla 2 y evidencian que el alumnado español obtiene las mejores medias estadísticas.

Tabla 2 - Estadísticos descriptivos generales de la aplicación del WAI

\begin{tabular}{lcccccccc}
\hline País & Media & Mediana & Máximo & Mínimo & Moda & Rango & Des. típica & Varianza \\
\hline España & 95,50 & 94,50 & 120,00 & 67,00 & 67,00 & 53,00 & 15,98 & 255,39 \\
\hline Finlandia & 85,88 & 81,50 & 109,00 & 72,00 & 77,00 & 37,00 & 12,65 & 160,13 \\
\hline Dinamarca & 89,63 & 85,50 & 118,00 & 65,00 & 105,00 & 53,00 & 18,31 & 335,41 \\
\hline
\end{tabular}

Fuente: Elaboración propia

Con respecto al análisis por sexos, tanto los alumnos finlandeses como los daneses obtienen mejores resultados que las alumnas de sus respectivos países. Esta cuestión es opuesta en el caso español, donde las alumnas superan claramente a los alumnos, tal y como se recoge en la Tabla 3 .

Tabla 3 - Estadísticos descriptivos por país y sexo en la aplicación del WAI

\begin{tabular}{l|c|c|c|c|c|c|c|c|c|c}
\hline País & \multicolumn{1}{c}{ Sexo } & \multicolumn{1}{c}{ Media } & \multicolumn{1}{c}{ Mediana } & \multicolumn{1}{c}{ Máximo } & \multicolumn{1}{c}{ Mínimo } & \multicolumn{1}{c}{ Moda } & \multicolumn{1}{c}{ Rango } & \multicolumn{2}{c}{ Desv. típica } & \multicolumn{1}{c}{ Varianza } \\
\hline \multirow{2}{*}{ España } & Hombre & 88,50 & 83,50 & 120,00 & 67,00 & 67,00 & 53,00 & 22,84 & 521,67 \\
& Mujer & 100,17 & 97,00 & 116,00 & 92,00 & 92,00 & 24,00 & 9,02 & 81,37 \\
\hline \multirow{2}{*}{ Finlandia } & Hombre & 93,00 & 93,00 & 109,00 & 77,00 & 77,00 & 32,00 & 22,63 & 512,00 \\
& Mujer & 83,50 & 81,50 & 98,00 & 72,00 & 72,00 & 26,00 & 9,73 & 94,70 \\
\hline \multirow{2}{*}{ Dinamar- } & Hombre & 93,67 & 95,50 & 118,00 & 65,00 & 105,00 & 53,00 & 19,20 & 368,67 \\
& Mujer & 77,50 & 77,50 & 85,00 & 70,00 & 70,00 & 15,00 & 10,61 & 112,50 \\
\hline
\end{tabular}

Fuente: Elaboración propia 
Los resultados de las seis escalas que incorpora el WAI proporcionan una interesante información global del alumnado participante, su potencial, intereses y actitudes hacia el trabajo. En la Gráfica 1, se muestra la representación de las Escalas en función de cada uno de los países.

Gráfica 1 - Representación de las Medias obtenidas escala/país

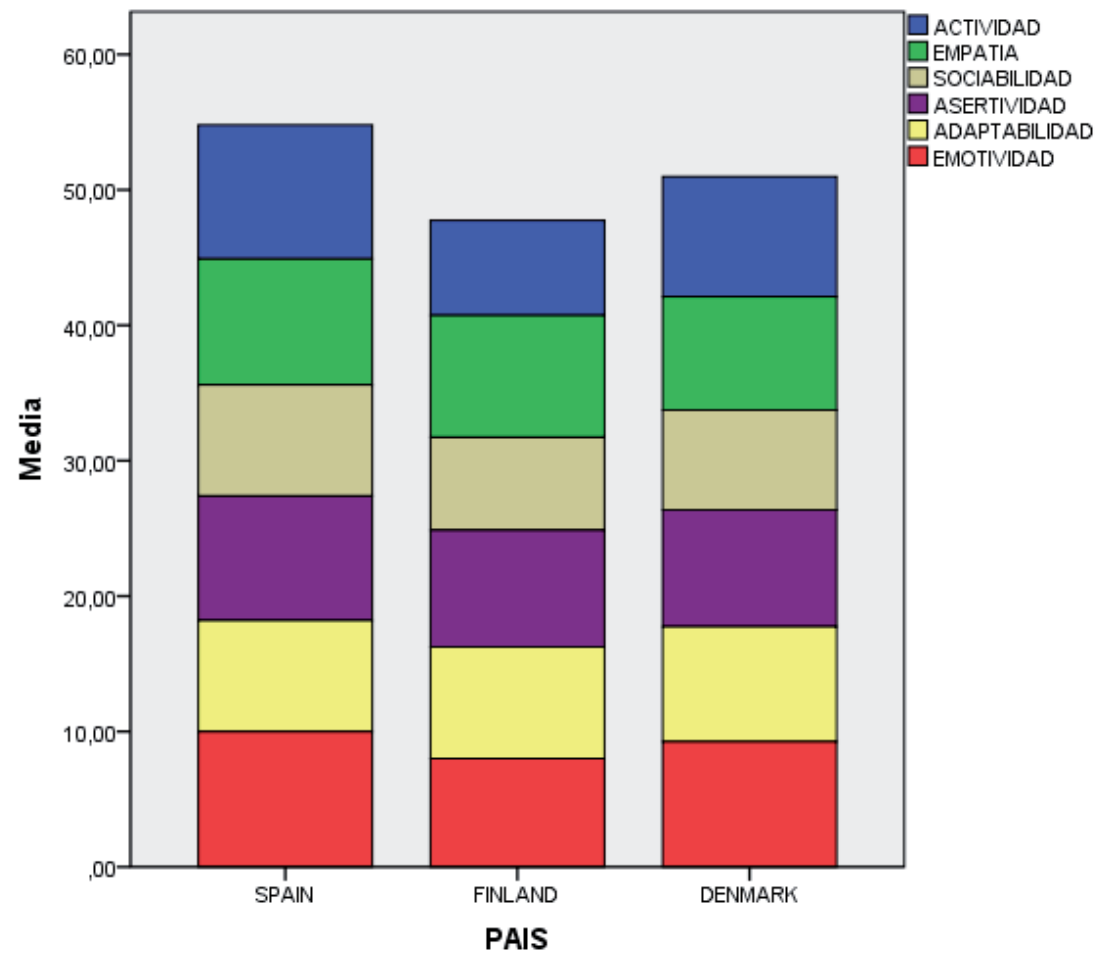

Fuente: Elaboración propia

El alumnado español muestra las medias más altas en cinco de las escalas (actividad, empatía, sociabilidad, asertividad y emotividad), no así en adaptabilidad, en donde el alumnado danés ha puntuado más alto.

El alumnado finlandés presenta puntuaciones más bajas que los daneses en cuatro de las escalas (adaptabilidad, emotividad, actividad y sociabilidad), no así en empatía (claramente superiores) y en asertividad (que están en igualdad de condiciones).

En la escala de actividad el alumnado de Finlandia presenta puntuaciones más bajas que los daneses, lo que nos lleva a pensar que este alumnado muestra menor interés en trabajar regularmente o estar ocupados. Pueden tener problemas para mantenerse en la tarea y pueden empezar trabajos y no terminarlos. Personas que tienen puntuaciones bajas pueden tener problemas con la asistencia, puntualidad o normas impuestas por otros. 
En la escala de empatía ha sido el alumnado danés el que ha puntuado más bajo, lo que nos lleva a pensar que estamos ante estudiantes más reservados, que prefieren guardar sus opiniones. Asimismo, los estudiantes daneses han obtenido puntuaciones más altas en la escala de emotividad que en las demás escalas.

Posteriormente, se procedió a establecer correlaciones entre las distintas escalas del WAI, entendiéndolas como nivel de asociación entre escalas para analizar si podrían identificarse influencias entre unas y otras. Los datos obtenidos muestran que la escala sociabilidad tiene una alta correlación $(\mathrm{p}<.001)$ con la escala actividad y empatía, y viceversa. Asimismo, la escala Adaptabilidad está relacionada con las escalas de actividad, empatía, sociabilidad y asertividad.

Gráfica 2 - Representación de correlaciones entre escalas

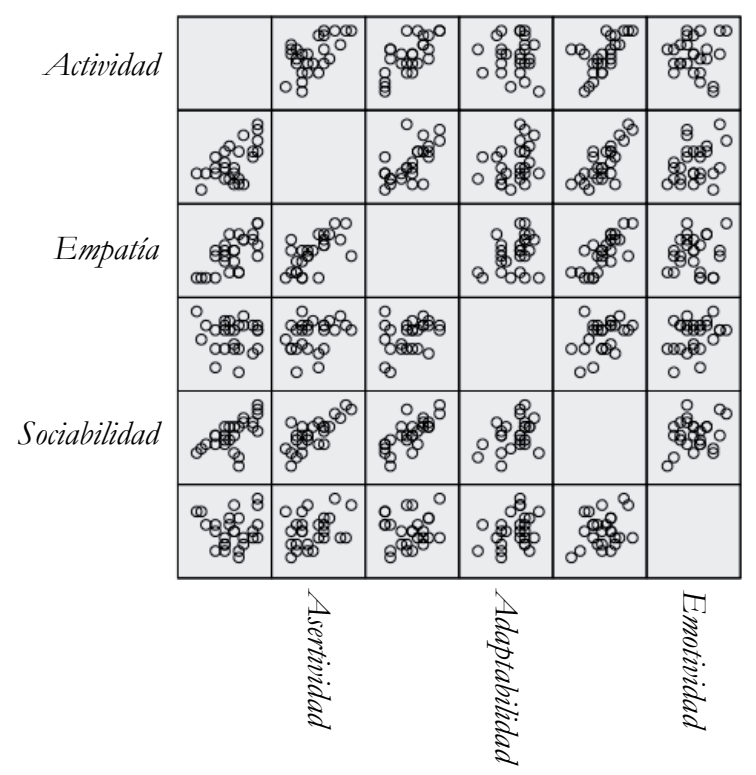

Fuente: Elaboración propia

Todas las escalas del WAI están fuertemente correlacionadas de una u otra forma, por lo que no deben trabajarse de forma aislada dentro del proceso formativo del alumnado cuando el objetivo es conseguir su óptimo desarrollo integral como personas preparadas para una inserción social y laboral acorde a sus posibilidades.

\subsection{LAS EXPECTATIVAS DE LAS FAMILIAS}

Los principales resultados obtenidos mediante las entrevistas familiares realizadas ponen de manifiesto que el $70 \%$ de los jóvenes participantes son los primeros hijos de la unidad familiar (un 30\% de los cuales son hijos únicos y el resto tienen un hermano menor); mientras que el 30\% restante son los menores (de 2 ó incluso 3 hermanos).

El diagnóstico de la discapacidad fue realizado a los pocos meses de vida (20\%), al año (10\%), entre 3 y 4 años (60\%) y/o en secundaria (10\%). Al preguntar por el tipo de 
especialista al que acudieron cuando fueron conscientes de la problemática de su hijo, fueron varias las respuestas, pero se decantaron en la misma proporción (20\%) a distintos profesionales: pediatra, médico de familia, psicólogo, gabinetes privados y los servicios facilitados por el centro escolar en que se encontraba en ese momento.

La reacción de las familias (Gráfica 3) al conocer la situación de discapacidad de su hijo/a ha sido diferente según los casos, aunque la más común es la de sentirse mal o estar muy afectados, si bien en la mayoría de los casos estos sentimientos se han ido superando con los años (Madre 2). Sin embargo, una madre confesó que aún estaba con depresión y que no conseguía asimilar que su hija con lo guapa que es, no sea como los demás (Madre 7). Si categorizamos la información obtenida comprobamos que el $40 \%$ de las familias manifiesta que la reacción fue negativa por la noticia, si bien, un 30\% manifestó que reaccionaron bien ante tales situaciones. Gráfica 3 - Categorías. Reacción de las familias

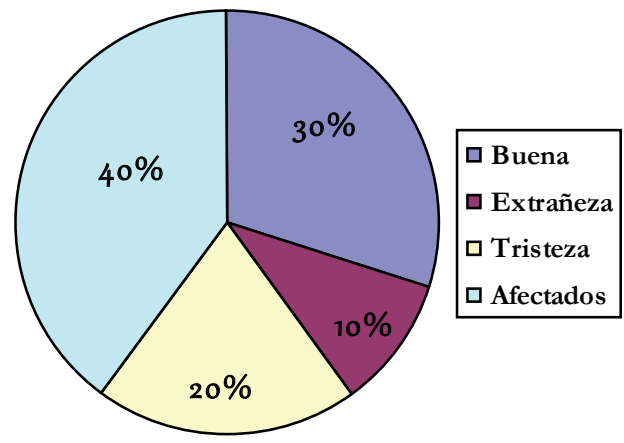

Fuente: Elaboración propia

Es reseñable que el mismo porcentaje de familias (40\%) que manifiesta su malestar cuando son conscientes de las limitaciones de su hijo/a, se siente satisfecho cuando se propone a sus hijos/as que la formación se oriente a programas específicos de inserción laboral que se desarrollan en centros especiales, como es el caso de los PCPI. Además un 30\% de las familias manifiesta que era algo que veniamos demandando desde la educación primaria (Madre 3). Por el contrario, un 20\% indica su malestar cuando fueron informados de que la mejor opción para sus hijos era un centro específico de educación especial, pero aún así lo aceptaron, y a día de hoy un 90\% manifiesta estar muy contenta con la formación que recibe mi hijo (Madre 7).

En lo referido a la pregunta ¿Cómo ve la familia a los jóvenes? el $60 \%$ de las familias entrevistadas coinciden en señalar que su hijo/a es vago, distraido o con falta de iniciativa (Madre 5). Esa información aporta un dato relevante que señala que las familias aún no son conscientes completamente de la problemática real de sus respectivos hijos/as (Gráfica 4). 
Gráfica 4 - Categorías. Dificultades y virtudes

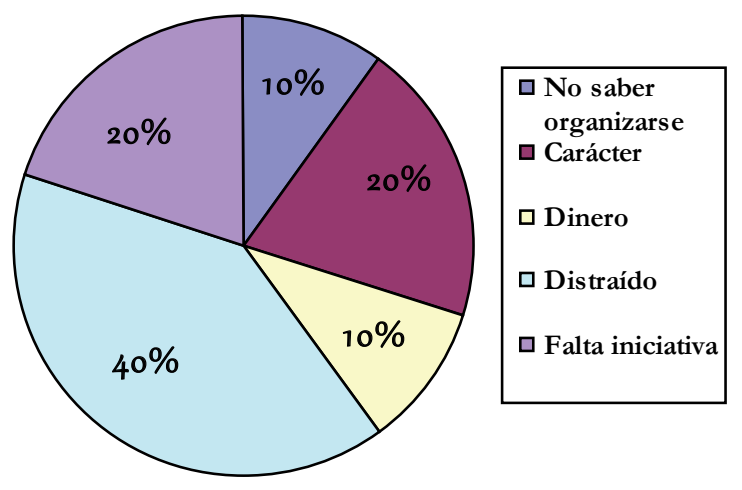

Fuente: Elaboración propia

En cuanto a las virtudes, las familias también coinciden al señalar los rasgos de personalidad como los elementos más positivos de sus hijos/as (80\%). Así por ejemplo algunas madres manifestaban que la bondad, la nobleza y la tranquilidad de su hija (Madre 16) eran los elementos más destacables. Dado que estas familias han tenido que vivir su realidad desde el momento del diagnóstico, nos interesaba saber qué habían aprendido emocionalmente de su día a día con estos jóvenes con discapacidad (Gráfica 5). Los datos revelan que la paciencia ha sido uno de los elementos más reiterados en las respuestas aportadas por las familias como elemento fundamental que define su aprendizaje emocional.

Gráfica 5 - Categorías. Aprendizaje emocional

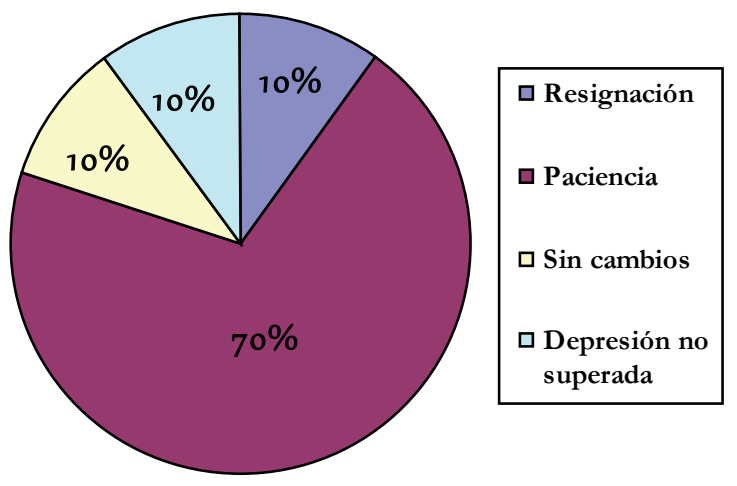

Fuente: Elaboración propia

Para orientar el tiempo libre del alumnado era preciso conocer qué tipo de actividades realizaban habitualmente, con quién salía, si tenía amigos, etc. El 60\% de los participantes no tiene amigos y si los tiene suelen ser más pequeños, por lo que con el tiempo los terminan perdiendo. En su tiempo libre sólo un 20\% sale con amigos, mientras el otro $80 \%$ se queda en casa delante del TV o jugando con Internet (en un 50\% de los casos) o sale con sus padres (un 
20\%). De los que se quedan en casa, el 70\% están continuamente con la madre acompañándola en lo que haga y un 30\% están haciendo lo que quieren en todo momento (Madre 13). Dado que la actividad principal se circunscribe al ámbito del domicilio familiar, se preguntó por el tipo de actividades domésticas que realizaban, destacando que el 60\% del alumnado realizaba todo tipo de actividades domésticas.

En cuanto a las perspectivas de futuro, se plantearon preguntas sobre tres momentos diferentes: 1. en un futuro inmediato al terminar el programa formativo de inserción laboral; 2. dentro de la horquilla de 3 a 5 años cuando ellos -como progenitores-ya no estuvieran. En este último caso debemos decir que un $60 \%$ de las madres confesaron no haberse planteado nunca este supuesto.

Tal y como se recoge en el Gráfica 6, un 50\% de las familias espera que sus hijos/ as puedan incorporarse al mundo laboral cuando terminen el programa, pues entienden que estarán lo suficientemente preparados, mientras que un $20 \%$ no tienen nada claro a este respecto.

Gráfica 6 - Categorías. Opiniones futuro inmediato

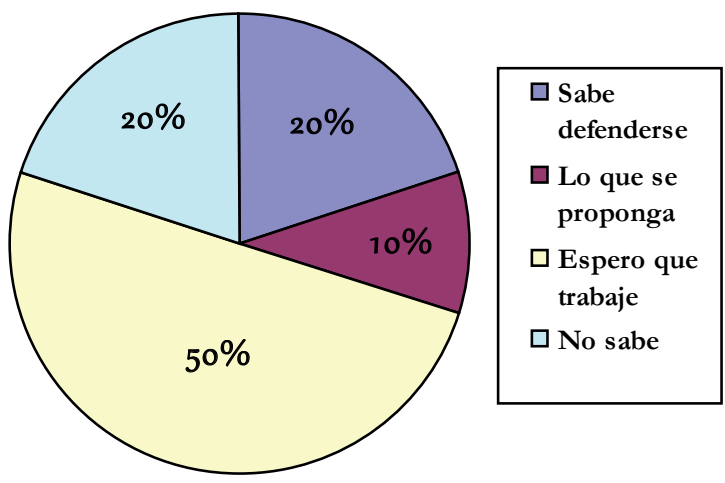

Fuente: Elaboración propia

Sin embargo parece que las opiniones se van modificando y, de esta forma, cuando la pregunta se enfoca como expectativa en un plazo de cinco ańos, un $60 \%$ de las familias cree que sus hijos estarán trabajando; algunos con pareja (20\%), y otros viviendo de forma independiente (10\%) o con ellos (30\%). En cualquier caso, la idea de inserción laboral predomina, ante un 30\% que sigue viéndolos dependientes de la familia en todos los sentidos, o un $10 \%$ que sigue sin plantearse nada.

Dado que el 60\% de familias consideraba que sus hijos estarían trabajando en un plazo de cinco años, se les preguntó si les ofrecerían trabajo en caso de ser ellos empresarios. A este respecto, un $80 \%$ contestó de forma afirmativa defendiendo la idea que pueden desempeńar la misma labor que cualquier otra persona, aunque necesiten ser supervisados. Por el contrario un $20 \%$ consideró que no están lo suficientemente preparados para incorporarse al mundo laboral. 
Al preguntarles por el tipo de trabajo que podrían desempeñar estos jóvenes con discapacidad (Gráfica 7), un 50\% de las familias considera que pueden insertarse laboralmente en funciones auxiliares; un $20 \%$ en cualquier actividad que no requiera responsabilidad, un $10 \%$ consideró que como celadores, otro $10 \%$ lo vio preparado para cualquier cosa y, finalmente, un 10\% no planteó ninguna posibilidad sobre esta cuestión.

Gráfica 7 - Categorías. Posibilidades laborales

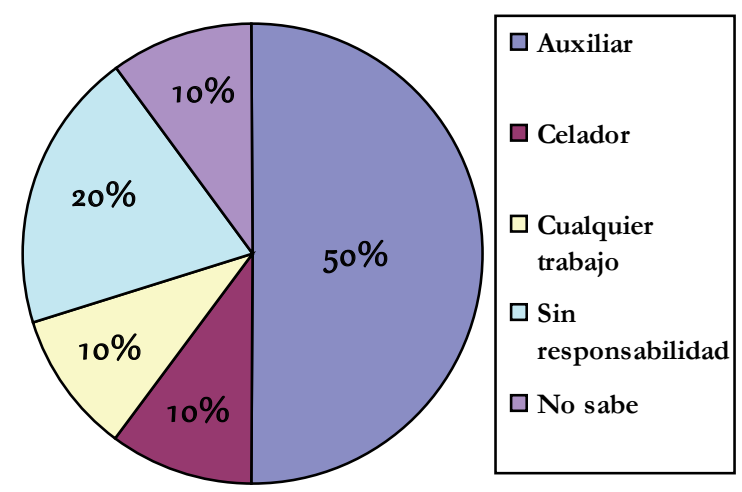

Fuente: Elaboración propia

Durante las entrevistas también se consultó sobre la expectativa atribucional que según las familias tiene la sociedad hacia las personas con discapacidad, de forma general y en relación a sus hijos e hijas en particular. A este respecto, el 70\% considera que los sentimientos de lástima son la manifestación principal que la sociedad tiene hacia este colectivo, frente a un $20 \%$ de las familias que manifestó la aceptación plena y un $10 \%$ que indicó que la sociedad continúa rechazándolos. Pero al focalizarlo en su propio hijo, la lástima bajó a un $10 \%$, aumentando a un 50\% el rechazo y los problemas con familiares, y estando un $40 \%$ en situación de aceptación, o no identificándose con ninguna, pues según argumentaron no han percibido nada extraño.

\section{Conclusiones y discusión}

Los datos cuantitativos que se han obtenido mediante el Work Adjustment InventoryWAI aplicado al alumnado con discapacidad participante y la información de carácter cualitativa proporcionada por las familias a través de las entrevistas realizadas nos permiten afirmar que el itinerario de inserción sociolaboral diseñado constituye una oferta formativa que se ajusta a las características del alumnado destinatario potencial. Asimismo las expectativas familiares sobre la futura inserción laboral que tienen las familias de alumnado muestran una confianza mayoritaria en las competencias de sus hijos/as y en las posibilidades que el itinerario formativo de inserción sociolaboral puede tener para mejorarlas e incrementarlas. De esta forma hemos logrado orientar este estudio y los resultados obtenidos a la consecución de los dos objetivos previstos inicialmente. 
Tras el análisis del perfil del alumnado implicado en los centros europeos participantes, podemos concluir que existen altas correlaciones entre la sociabilidad del alumnado y su grado de actividad y empatía. Asimismo, la información recabada nos permite avanzar que el grado de adaptabilidad del alumnado está relacionado con el nivel de actividad, empatía, sociabilidad y asertividad que posea. Por tanto, cuanto más sociable sea un estudiante más interés mostrará por el trabajo y por estar ocupado, y será capaz de ponerse en el lugar del compañero ante un problema. En otras palabras, buenas puntuaciones en Actividad, Empatía, Sociabilidad, Asertividad, Emotividad y Adaptabilidad suponen una coherencia interna en las personas que no solo se concretan en el trabajo, sino en aspectos familiares, culturales y/o sociales de la persona, creándose un vínculo entre el trabajo y las demás dimensiones de la vida por lo que la formación al respecto debe tener presente esta interrelación para su diseño, desarrollo y evaluación.

La información proporcionada por las entrevistas realizadas permite trazar un perfil de las familias que, en líneas generales, se vio profundamente afectada por la noticia inicial de tener un hijo/a con discapacidad pero que han ido desarrollando con el paso del tiempo una importante actitud de confianza en sus respectivos hijos. Así las familias identifican ciertas dificultades en sus hijos en cuanto al carácter y la organización en el ámbito del hogar. Esta cuestión está estrechamente relacionada con el tipo de aprendizaje emocional que las madres han venido desarrollando y que mayoritariamente se relaciona con la paciencia. Conocer las autopercepciones es una cuestión que consideramos fundamental pues coincidimos con Jurado y Olmos (2010), en la importancia de partir del análisis de la autopercepción de las competencias y del grado de ajuste al trabajo por parte de los estudiantes, tal y como plantean en su modelo, para lograr la inserción laboral.

En cuanto a las expectativas de futuro, tanto las familias como las puntuaciones del alumnado tienen aspectos coincidentes que se orientan hacia la identificación de habilidades profesionales y personales que les permitirán insertarse laboralmente de una manera adecuada. En este sentido las familias valoran altamente que se diseñe un itinerario personalizado de inserción para el alumnado con discapacidad con el objetivo de promover una transición a la vida activa coherente y eficaz desde la perspectiva de la planificación centrada en la persona y la autodeterminación. Coincidimos, por tanto, con las aportaciones de Martínez y Echeverría (2009) en la necesidad de cambiar el diseńo de los programas formativos y el rol de docentes y alumnado, así como con Vilá, Plallisera y Fullana (2012) en que es necesario que sean estos jóvenes los verdaderos protagonistas del itinerario que les facilite la inclusión sociolaboral. Se trata, por tanto, de promover el diseño de un proyecto profesional y vital individualizado, sin ceder a las presiones económicas, sociales y familiares, fijando como objetivo a la persona (FERNÁNDEZ, 2006).

La inserción sociolaboral se incrementa cuando el alumnado destinatario mejora sus competencias profesionales y sociopersonales tal como se prevé en el diseño del itinerario personalizado. En su desarrollo que, en el caso de España se implementa a través de un Programa de Cualificación Profesional Inicial (modalidad Taller especifico), constituye una medida que une el ámbito más académico con el laboral y profesional, enclavándose ahí gran parte de su relevancia defendida en estudios como Aramendi y Vega (2013) o Aramendi, Vega y Buján (2012) que plantean que los PCPI se constituyen como un recurso facilitador de la inclusión sociolaboral de jóvenes con discapacidad. 
Los datos obtenidos están en la línea de estudios recientes como los realizados por Marín, García y Sola (2014) sobre los PCPI, concluyendo que no sólo constituyen instrumentos para la obtención de competencias mínimas para insertarse en el ámbito laboral, sino que son espacios de convivencia y de relaciones pues el alumnado, el profesorado y otros profesionales participantes, desarrollan vínculos de gran valor. A este respecto, la información suministrada por las familias y también los datos del WAI están en la línea de las conclusiones de Vega y Aramendi (2013) que sitúan los PCPI como una medida altamente valorada por el alumnado participante, ya que el itinerario personalizado de inserción puede satisfacer al alumnado con discapacidad participante porque se encuentra estrechamente vinculada con el desarrollo de competencias relacionales, con el área laboral y, en su conjunto, con el desarrollo de un proyecto de vida inclusivo.

Por lo tanto, los PCPI con su carácter intrínseco de dinamización, adaptación al alumnado y educación integral real, se han de postular como garantía de inserción laboral y social, capaz de afrontar las necesidades generales de su alumnado, otorgando la importancia que tiene al ámbito de socialización y disminuyendo, por tanto, las dificultades de integración social (García-Ruiz, Sánchez y Rodríguez-Martín, 2013). De esta manera pueden responder a las acertadas demandas planteadas en diferentes estudios (López, Salmerón y Salmerón, 2010; Riaño, 2012), en los que se exponen la necesidad de relacionar el contexto educativo con su entorno y, en general, el valor que posee la adquisición de competencias sociales y ciudadanas para lograr un exitoso desempeño sociolaboral.

En cuanto a las limitaciones del estudio, la muestra es reducida y los resultados no permiten generalizar las opiniones recogidas aunque están en la línea de estudios previos. No obstante, consideramos que la información recabada sí es relevante para establecer la base de una línea de investigación en la que se recoja la voz de los propios implicados, en una muestra mucho mayor, de cara a mejorar el diseño de los curriculum de los PCPI (Taller específico) y mejorar así la adquisición de competencias, minimizando la dicotomía existente entre las percepciones de los estudiantes y las demandas del entorno laboral.

Como líneas de futuro, hubiese sido interesante complementar este estudio con entrevistas en profundidad al alumnado y al profesorado potencial que pueda participar en los itinerarios personalizados, e incluso contrastar las opiniones a través de grupos de discusión, si bien continuamos trabajando en esta línea. También sería importante desarrollar futuras investigaciones con muestras más amplias que permitieran confirmar la información obtenida así como analizar la formación específica del profesorado en este ámbito y reforzar la acción de los orientadores en el desarrollo de competencias sociolaborales. No obstante, los datos obtenidos en este estudio invitan a reflexionar sobre las posibilidades reales de inclusión sociolaboral de jóvenes con discapacidad y las opciones que bridan los itinerarios personalizados desde la planificación centrada en la persona que, en el caso del sistema educativo espańol, pueden implementarse adecuadamente en los aún vigentes Programas de Cualificación Profesional Inicial o en la Futura Formación Profesional Básica. 


\section{REFERENCIAS}

ARAMENDI, P.; VEGA, A. Los Programas de Cualificación Profesional Inicial. La perspectiva del alumnado del País Vasco. Revista de Educación, v.360, p.436-460, 2013

; BUJÁN, K. Los programas de diversificación curricular y los programas de cualificación profesional inicial ¿̨una alternativa al fracaso escolar? Revista española de pedagogía, v.70, n.252, p.237255,2012

BISQUERRA, R. Metodología de la investigación educativa. Madrid: La Muralla. 2004.

CALERO, J.; CHOI, A.; WAISGRAIS, S. Determinantes del riesgo de fracaso escolar en Espańa: una aproximación a través de un análisis logístico multinivel aplicado a PISA-2006. Revista de Educación, número extraordinario, 225-256, 2010.

CARNABY, S.; LEWIS, P. Involving young people with learning disabilities leaving school in planning for the future. En CAMBRIDGE, P.; CARNABY, S. Person centred planning and care management with people with learning disabilities. London: Jessica Kingsley, 2005, p.84-99.

COOK, T.; REICHARDT, CH. Métodos Cuantitativos y Cualitativos en investigación Educativa. Madrid: Morata, 2005.

ECKERT, E. Entre el fracaso escolar y las dificultades de inserción profesional: la vulnerabilidad de los jóvenes sin formación en el inicio de la sociedad del conocimiento. Revista de Educación, v.341, p. 35-55, 2006.

FERNÁNDEZ, C.R. La integración laboral de las personas con discapacidad. Temas para el debate, v.102, p.30-34, 2003.

FLICK, U. Introducción a la investigación cualitativa. Madrid: Morata, 2004.

FOX, D. El proceso de investigación en educación. Pamplona: Eunsa, 1981

FRANKLIN, C.; BALLAU, M. (2005). Reliability and validity in qualitative research. En GRINNELL, R.; UNRAU, Y. Social work: Research and evaluation. Quantitative and qualitative approaches. Nueva York: Oxford University Press, 2005, p. 438-449.

GARCÍA-RUÍZ, R.; SÁNCHEZ, A.; RODRÍGUEZ-MARTÍN, A. Las competencias socio-personales en la integración socio-laboral de los jóvenes que cursan P.C.P.I.. Revista Española de Orientación y Psicopedagogía, v.24, n.2, p.58-78, 2013.

GILLIAM, J. WAI: Work Adjustment Inventory: Measures of job-related temperament. Austin, Texas: ProEd, Inc., 1994.

GOROSTIDI, M.; NAVARRO, G. Inserción sociolaboral para los discapacitados: El empleo con apoyo. En IPLAND J. et al. Atención a la diversidad, una responsabilidad compartida. Huelva: Servicio de Publicaciones de la Universidad de Huelva, 2007.

JURADO, P.; OLMOS, P. Procesos de orientación para la inserción sociolaboral de personas con especiales dificultades de acceso al mercado de trabajo. Un modelo de orientación para la inserción. Revista Española de Orientación y Psicopedagogía, v.21, n.2, p.93-108, 2010.

LEÓN, M.J. Retomando la educación Secundaria y buscando la incorporación al mundo laboral y a la vida adulta. Los Programas de Garantía Social como alternativa. En BUENO, J. et al. Atención Educativa a la diversidad en el nuevo milenio. A Coruña: Servicio de Publicaciones de la Universidad de la Coruña, 2001. p.363-378 
LÓPEZ, R.; SALMERÓN, P.; SALMERÓN, C. Desarrollo y evaluación de la competencia social y ciudadana en educación inclusiva. Efectos del aprendizaje cooperativo. Revista de educación inclusiva, v.3, n.2, p.29-46, 2010.

MARÍN, J.A.; GARCÍA, M.; SOLA, J.M. Reflexión y análisis sobre los Programas de Cualificación Profesional Inicial como medida de inclusión social y educativa en Andalucía (España). REICE. Revista Iberoamericana sobre Calidad, Eficacia y Cambio en Educación, v.12, n.1, p.83-102, 2014.

MARTÍNEZ, P.; ECHEVERRÍA, B. Formación basada en competencias. Revista de Investigación Educativa, v.27, n.1, p.125-147, 2009.

MERINO, R.; GARCÍA, M.; CASAL, J. De los Programas de Garantía Social a los Programas de Cualificación Profesional Inicial. Sobre perfiles y dispositivos locales. Revista de Educación, v.341, p.8198, 2006.

MERTENS, D. Research and evaluation in Education and Psychology: Integrating diversity with quantitative, qualitative, and mixed methods. London: Sage Publications, 2005.

MILES, M.; HUBERMAN, M Qualitative Data Analysis: An Expanded Sourcebook. Beberly Hills: SAGE Publications, 1994.

MONTES GUIDOX, M. Conciliación laboral y personas con discapacidad. En IPLAND J. et al. Atención a la diversidad, una responsabilidad compartida. Huelva: Servicio de Publicaciones de la Universidad de Huelva, 2007.

PALLISERA, M. Apoyando proyectos de vida inclusivos: claves para transformar las prácticas socioeducativas de personas adultas con discapacidad intelectual. Revista de educación inclusiva, v.3, n.3, p.69-88, 2010.

;VILÀ, M.; VALLS, M.J. The Current Situation of Supported Employment in Spain: analysis and perspectives based on the perception of professionals. Disability \& Society, v.18, n.6, p.797-810, 2003.

RIAÑO, A.M. Programa de cualificación inicial. Itinerario laboral y planificación centrada en las personas con necesidades diversas. Siglo Cero, v.43, n.4, p.5-20, 2012.

SANAHUJA, J.; NÚÑEZ, D.; JURADO, P. Algunos apuntes sobre el empleo con soporte. Incidencia en una proyecto específico. En BUENO, J.; NÚÑEZ, T.; IGLESIAS, A. Atención educativa a la diversidad en el nuevo milenio: XVIII Jornadas de Universidades y Educación Especial. La Coruña: Universidad de la Coruña, 2001. p.305-315

SÁNCHEZ, A.; JURADO, P. Los Programas de Garantía Social (PGS): Claves para su implantación. En BUENO, J.; NÚNEEZ, T.; IGLESIAS, A. Atención educativa a la diversidad en el nuevo milenio: XVIII Jornadas de Universidades y Educación Especial. La Coruña: Universidad de la Coruña. 2001, p. $507-523$

SCHALOCK, R.L. Integrando el concepto de calidad de vida y la información de la Escala de Intensidad de Apoyos en planes individuales de apoyo. Siglo Cero, v. 245, p. 6-21, 2013.

STAINBACK S.B.; STAINBACK W.C. Aulas inclusivas. Madrid: Narcea, 1999.

STAKE, R.E. Qualitative case studies. In DENZIN, N.K.; LINCOLN Y.S. Strategies of qualitative inquiry. Los Angeles: Sage Publications, 2008. p.119-149.

SUÁREZ, N. et al. El fracaso escolar en Educación Secundaria. Análisis del papel de la implicación familiar. Magister. Revista miscelánea de investigación, v. 24, p.49-64, 2011. 
TAYLOR, S.; BOGDAN, R. Introducción a los métodos cualitativos de investigación. Barcelona: Paidós, 1990.

VEGA, A.; ARAMENDI, P. Entre el fracaso y la esperanza. Necesidades formativas del alumnado de los Programas de Cualificación Profesional Inicial. Educación XX1, v.13, n.1, p.39-63, 1010.

. Los Programas de Cualificación Profesional Inicial. La perspectiva del alumnado del País Vasco. Revista de Educación, v. 360, 2010 (En prensa).

VERDUGO, M.A.; JORDÁN, B. Hacia una nueva concepción de la Discapacidad. Madrid: Siglo XXI, 1999.

VILÀ, M.; PALLISERA, M. Acciones a desarrollar desde el centro educativo para facilitar los procesos de inserción laboral de las personas con discapacidad: aportaciones a partir de una investigación. Bordón, v.1, p.103-116. 2006.

; FULLANA, J. La inclusión laboral de los jóvenes con discapacidad intelectual: un reto para la orientación psicopedagógica. Revista Española de Orientación y Psicopedagogía, v.23, n.1, p. 85-93, 2012. 
RIAÑO-GALÁN, A. et al. 